\title{
La amenaza del dengue, un riesgo real para Chile. Propuesta de nueva estrategia para su control
}

\section{The threat of dengue, a real risk for Chile. Proposal of a new strategy for its control}

\author{
Aldo Andrés Díaz Sepúlveda ${ }^{1}$
}

Palabras clave: brotes de enfermedades; dengue; Aedes aegytis; artrópodos; control de vectores; drogas veterinarias.

Keywords: disease outbreaks; dengue; Aedes aegyptis; arthropods; vector control; veterinary drugs.

Fecha de envío: 2020-09-29 - Fecha de aceptación: 2021-03-16

Señor editor:

Sin duda la pandemia por COVID-19 ha mantenido la atención de todo el mundo dado la magnitud de la situación y las consecuencias de la misma, no obstante, resulta necesario mantenernos alerta en todos los flancos interviniendo oportunamente. Así como hace mención el Dr. Mauricio Canals (2020) en la editorial titulada "Modelos epidemiológicos y la pandemia COVID-19 en Chile": la globalización, los cambios demográficos, ambientales y climáticos mantienen latente la aparición de enfermedades emergentes y re-emergentes, así como una progresiva expansión de las mismas en las zonas ya afectadas. Es así como previo al inicio de la pandemia por COVID-19, la afección por dengue representaba una de las enfermedades infecciosas más importantes en América Latina y el Caribe, alcanzando inclusive el máximo registro histórico en la notificacion de casos en la región y a nivel mundial durante el año 2019 (Organización Mundial de la Salud, 2020). Preocupa además en dengue la falta de medidas que impacten de forma significativa y sostenida en el tiempo, junto a la ausencia de tratamientos específicos y de vacunas efectivas, seguras, y de uso amplio, puesto que, la actual vacuna disponible solo está considerada en un subgrupo de la población ya expuesta al virus para la prevención de dengue grave.

El dengue ha mostrado un aumento sostenido en su incidencia en el mundo en las últimas seis décadas (Eby \& Nealon, 2016; Messina et al., 2019), misma tendencia que se observa en zonas tropicales y subtropicales de las Américas. De igual manera se ha extendido la distribución de los vectores pese a la implementación por la Organización Panamericana de la Salud de la estrategia de gestión integrada en la región. A nivel local preocupan los hallazgos en los últimos años en el norte de Chile de uno de los vectores, la especie Aedes aegyptis en Arica el año 2016 y en lquique 2019, junto a los conocidos brotes y casos de dengue con nuevos serotipos en Rapa Nui desde el año 2002 a la fecha (Ministerio de Salud, 2020). Si bien ha predominado el serotipo DEN-1 en esta región de Pacífico, se han presentado casos de DEN-2 y DEN-4 en Isla de Pascua.

Entendiendo la complejidad multifactorial del dengue y otras arbovirosis, y la necesidad de la participación interdiciplinaria tanto en la prevención como en el manejo, es que resulta importante buscar y generar nuevas herramientas para ello. En esta búsqueda han aparecido opciones para el control de la población de los vectores tales como el uso de radiación ionizante en mosquitos, o liberación de estos genéticamente modificados, así como, el uso de bacterias del género Wolbachia como control biológico, entre otros que están en aún en evaluación (Ache et al., 2019). En este sentido también es alentador la posible utilidad de las isoxazolinas, grupo de antiparasitarios ampliamente utilizados en la medicina veterinaria y más cercanamente en el cuidado de nuestras mascotas, siendo el ejemplo más conocido el fármaco fluralaner (Bravecto ${ }^{\circledR}$ de Merck Sharp \& Dohme); destaca en las características de este grupo de fármacos el efecto inhibidor potente y selectivo sobre los receptores ácido $\gamma$-aminobutírico $(\mathrm{GABA})$ y receptores del

(1) Hospital de Hanga Roa, Isla de Pascua, Región de Valparaíso. Chile Autor de correspondencia: aldo.diaz@hospitalhangaroa.cl 
glutamato en el sistema nervioso de los artrópodos (Gassel et al., 2014) tales como pulgas y garrapatas, así como mosquitos del género Anopheles, Aedes y Culex (Jiang et al., 2017; Miglianico et al. 2018).

Basados en la selectividad de la droga por los receptores de ácaros e insectos por sobre los de los mamíferos (Gassel et al., 2014; Miglianico et al., 2018), los amplios estudios sobre seguridad en animales, la farmacocinética que permite que una dosis mantenga niveles plasmáticos adecuados por semanas (Miglianico et al., 2018), propuestas de modelamientos matemáticos de efectividad en el manejo de arbovirosis, malaria, leishmaniasis como lo plantea Miglianico et al.(2018) en el artículo "Repurposing isoxazoline veterinary drugs for control of vector-borne human diseases" en la revista Proceedings of the National Academy of Science of the United State of America; e incipiente usos favorables en humanos como el realizado por Goldust en Escabiosis y presentado el año 2018 en el congreso anual de la Academia Americana de Dermatología en San Diego, California, es que resulta estimulante plantear el emprendimiento de mayores estudios para su utilización, ya sea para un uso periódico y masivo en comunidades con alta prevalencia de la enfermedad, expuestas a estos vectores con el fin de reducir la población de mosquitos y por tanto el riesgo de brote; o en un escenario clínico para la administración en pacientes sospechosos de dengue en fase febril (viremia) de lugares endémicos y/o donde exista riesgo de brotes dada la presencia del vector como es el caso Isla de Pascua o el norte de Chile. Efectos que sin duda deberán determinarse en estudios clínicos, para así ser considerado como nueva herramienta preventiva y poder cerrar uno de los cabos de la cadena de transmisión; todo esto en la suma de acciones para la eliminación de estas infecciones transmitidas a los seres humanos por artrópodos con la potencialidad de evolucionar grave clínicamente y lograr quebrar su tendencia.

Cordialmente,

Fuentes de financiamiento: Ninguna.

Declaración de conflictos: El autor declara no tener conflicto de interés.

\section{Referencias}

Achee NL, Grieco JP, Vatandoost H, Seixas G, Pinto J, Ching-Ng, L, Martins, AJ, Juntarajumnong, W, Corbel V, Gouagna, C, David JP, Logan JG, Orsborne J, Marois E, Devine GJ. \& Vontas, J. (2019). Alternative strategies for mosquito-borne arbovirus control. PLOS neglected tropical diseases 13, e0006822.
Canals LDM. (2020). Modelos epidemiológicos y la pandemia COVID-19 en Chile: Epidemiological models and the COVID-19 pandemic in Chile. ARS MEDICA Revista De Ciencias Médicas 45, 3-4. Ebi KL. \& Nealon J. (2016). Dengue in a changing climate. Environmental research 151, 115-123.

Gassel M, Wolf C, Noack S, Williams H. \& Ilg T. (2014). The novel isoxazoline ectoparasiticide fluralaner: selective inhibition of arthropod $\gamma$-aminobutyric acid- and L-glutamate-gated chloride channels and insecticidal/acaricidal activity. Insect biochemistry and molecular biology 45, 111-124.

Goldust M. (2018). Oral Ivermectin vs. Oral Fluralaner for the Treatment of Scabies. Abstract 6533. In: American Academy of Dermatology Annual Meeting. San Diego, CA.; 2018.

Jiang S, Tsikolia M, Bernier UR. \& Bloomquist JR. (2017). Mosquitocidal Activity and Mode of Action of the Isoxazoline Fluralaner. International journal of environmental research and public health 14, 154.

Organización Mundial de la Salud. (2020). Dengue y dengue grave. Accedido en: https://www.who.int/es/news-room/fact-sheets/detail/ dengue-and-severe-dengue el 29 de agosto de 2020

Messina JP, Brady OJ, Golding N, Kraemer M, Wint G, Ray SE, Pigott DM, Shearer FM., Johnson K, Earl L, Marczak LB, Shirude S, Davis Weaver N, Gilbert M, Velayudhan, R, Jones P, Jaenisch T, Scott TW, Reiner RC Jr. \& Hay SI. (2019). The current and future global distribution and population at risk of dengue. Nature microbiology 4 , 1508-1515.

Miglianico $\mathrm{M}$, Eldering $\mathrm{M}$, Slater $\mathrm{H}$, Ferguson $\mathrm{N}$, Ambrose $\mathrm{P}$, Lees RS, Koolen K, Pruzinova K, Jancarova M, Volf P, Koenraadt C, Duerr HP, Trevitt G, Yang B, Chatterjee AK, Wisler J, Sturm A, Bousema T, Sauerwein RW, Schultz PG. et al. (2018). Repurposing isoxazoline veterinary drugs for control of vector-borne human diseases. Proceedings of the National Academy of Sciences of the United States of America 115, E6920-E6926.

Ministerio de salud. (2020). Boletín Epidemiológico Trimestral: Arbovirus (Dengue, Zika, Chikungunya, Fiebre amarilla), SE 1 - 52, año 2019. Accedido en: http://epi.minsal.cl/wp-content/ uploads/2020/02/BET_ARBOVIROSIS_AÑO_2019-1.pdf el 16 de agosto de 2020 . 